\title{
A bio-inspired 3-DOF light-weight manipulator with tensegrity $X$-joints*
}

\author{
Benjamin Fasquelle, Matthieu Furet, Parag Khanna, Damien Chablat, \\ Christine Chevallereau, Philippe Wenger
}

\begin{abstract}
This paper proposes a new kind of light-weight manipulators suitable for safe interactions. The proposed manipulators use anti-parallelogram joints in series, referred to as $\mathrm{X}$-joints. Each $\mathrm{X}$-joint is remotely actuated with cables and springs in parallel, thus realizing a tensegrity one-degree-offreedom mechanism. As compared to manipulators built with simple revolute joints in series, manipulators with tensegrity $X$-joint offer a number of advantages, such as an intrinsic stability, variable stiffness and lower inertia. This new design was inspired by the musculosleketon architecture of the bird neck that is known to have remarkable features such as a high dexterity. The paper analyzes in detail the kinetostatics of a $\mathrm{X}$-joint and proposes a 3-degree-of-freedom manipulator made of three such joints in series. Both simulation results and experiment results conducted on a test-bed prototype are presented and discussed.
\end{abstract}

\section{INTRODUCTION}

Animal musculoskeletal systems offer remarkable dexterity and dynamic features that make them much more efficient than existing industrial manipulators. Much less studied than other animal models, the bird neck turns out to exhibit very interesting performances. In fact birds use their neck like an arm for every-day-life tasks such as cleaning and feeding. Moreover, they often use their neck for dexterous tasks interacting with the environment (e.g., a vulture picking food from a carcass) as well as for tasks demanding high force transmissions and accelerations (e.g., the woodpecker hitting a tree trunk). Accordingly, bird necks are an interesting source of bio-inspiration for designing new manipulators with enhanced performances. This work emerged from a collaborative project with biologists for the purpose of understanding the behavior of bird necks and building a robotic model. The concept of tensegrity has been chosen in this project. A tensegrity structure is an assembly of compressive elements (bars) and tensile elements (cables, springs) held together in equilibrium [1], [2]. Tensegrity is known in architecture and art for more than a century [3] and is suitable for modeling living organisms [4]. Tensegrity mechanisms have been more recently studied for their promising properties in robotics such as low inertia, natural compliance and deployability [5], [6], [7]. They are also interesting candidates to design locomotion systems [8], [9], [10], [11], [12]. A tensegrity mechanism is obtained when one or several elements are actuated. A class of planar tensegrity manipulators made of a series assembly

*This work was supported by the French National Research Agency, AVINECK Project ANR-16-CE33-0025

École Centrale de Nantes, CNRS, Laboratoire des Sciences du Numérique de Nantes (LS2N), UMR CNRS 6004, 1 rue de la Noë, 44321 Nantes, France ffirstname.name\}@ls 2 n. fr of several tensegrity $\mathrm{X}$-shape mechanisms i.e. crossed fourbar mechanisms with springs along their lateral sides, has been chosen as a suitable candidate for a preliminary planar model of a bird neck, see Fig. 1. These mechanisms, referred to as tensegrity X-joints, are inspired from the Snelson's Xshape mechanisms [13]. Although simplified because it is planar, this model goes beyond the only available bird neck model in the literature that uses a simple planar articulated linkage [14], as it can be more easily actuated with cables that play the role of tendons and muscles. Moreover, the center of rotation of the $\mathrm{X}$-joint is not fixed, which is the case in most biological joints. Snelson's X-shape mechanisms have been studied by a number of researchers, either as single mechanisms [5], [7], [15], [16] or assembled in series [17], [18], [12], [11], [19], [20], [21]. In this paper, the manipulator may be subject to gravity unlike in [11], where the mechanism was used in a snake-like manipulator moving on the ground.

This paper proposes a new paradigm for designing lightweight manipulators using tensegrity $\mathrm{X}$-joints. As compared to a simple revolute joint, the proposed joint offers a number of advantages, such as an intrinsic stability, variable stiffness and lower inertia. This paper analyzes in detail the kinetostatics of an X-joint and proposes a 3-degree-offreedom manipulator made of three such joints in series. A test-bed prototype is realized and described. Simulation and experiment results are presented and discussed.

\section{Single JOINT DESIGN AND MODELLING}

\section{A. Joint description}

A single tensegrity $\mathrm{X}$-joint is composed of a crossed fourbar linkage along with two lateral tension springs assumed mass-less. The base and top bars have the same lengths and so do the two crossed bars, thus defining a so-called antiparallelogram. The links are assumed infinitely rigid, and are connected with each other with perfect revolute joints (no friction or damping). The bottom bar defines the base of the X-joint. Unlike the original Snelson's X-shape tensegrity that has only two compressive elements (the two crossed bars) and four tensile elements (the four sides), the X-joints used in this paper have four bars in total. The two additional bars replace the tensile elements on the base and top sides. This choice has been mainly motivated by the need to keep torsion rigidity of the prototype that needs to be assembled in different layers to prevent self collisions (see Fig. 2). Accordingly, each X-mechanism used in this study is a class2 tensegrity [3]. 


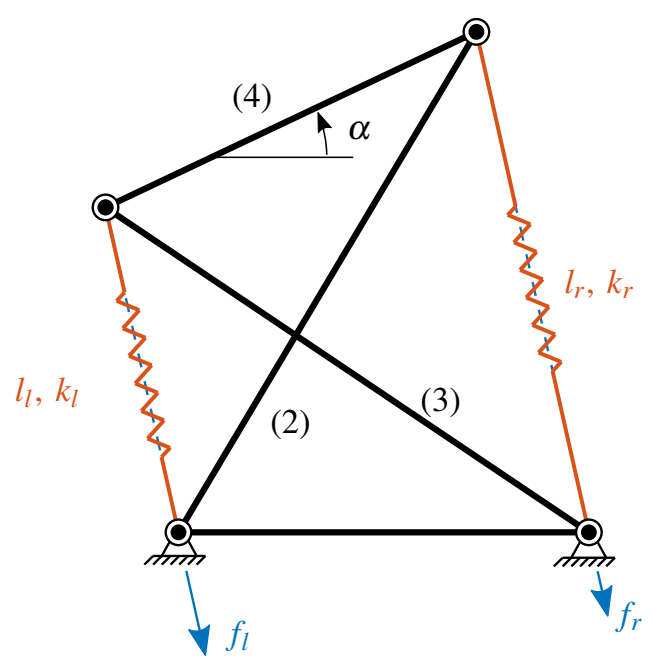

Fig. 1: Single tensegrity $\mathrm{X}$-joint.

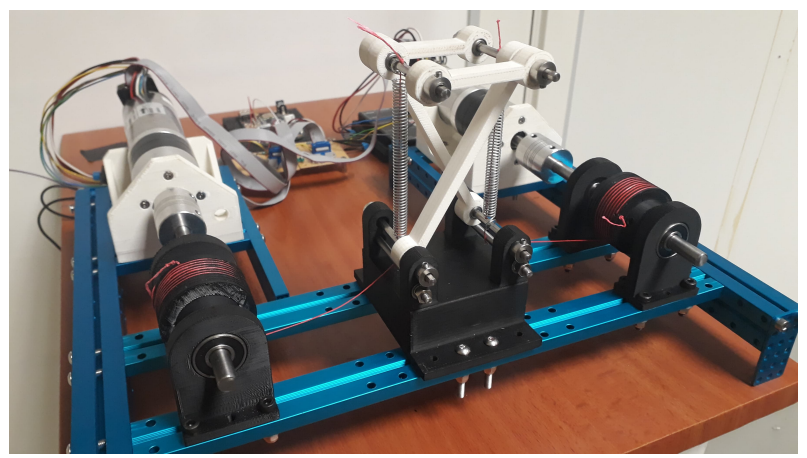

Fig. 2: Single X-joint testbed

The joint data are given in Tab. I. The two crossed bars (resp. the base and top bars) are of length $L$ (resp. $b$ ).

\section{B. Actuation scheme}

The X-joint is antagonistically actuated with two active cables running through the springs. Each cable is attached to a drum linked to a motor shaft. The cables are considered infinitely stiff. The radius $r_{d r u m}$ of the drums has been determined such that the maximal motor torque provides sufficient forces to actuate the $\mathrm{X}$-joint in its full range of rotation while keeping a satisfactory joint rate. Accordingly, the maximal tension force that the cables can apply is $f_{\max }=155 \mathrm{~N}$.

\begin{tabular}{ccc}
\hline Parameter & Value & Unit \\
\hline \hline$b:$ length of base and top bars & 0.05 & $\mathrm{~m}$ \\
$L:$ length of crossed bars & 0.1 & $\mathrm{~m}$ \\
$m_{4}:$ mass of one top bar & 0.009 & $\mathrm{~kg}$ \\
$m_{2}, m_{3}:$ mass of one crossed bar & 0.016 & $\mathrm{~kg}$ \\
$k_{l}, k_{r}:$ spring stiffness & 90 & $\mathrm{~N} / \mathrm{m}$ \\
$l_{0}:$ spring free length & 0.037 & $\mathrm{~m}$ \\
$r_{\text {drum }}:$ drum radius & 0.02 & $\mathrm{~m}$ \\
$I_{a}:$ Inertia of actuator at drive & 0.0078 & $\mathrm{~kg} \cdot \mathrm{m}^{2}$ \\
$f_{\max }:$ Maximal force applied by cable & 155 & $\mathrm{~N}$ \\
\hline
\end{tabular}

TABLE I: 1-DoF Prototype data.

\section{Dynamic model}

The $\mathrm{X}$-joint configuration is described by the angle $\alpha$ between the bottom and top bars. The rotation range is $-\alpha_{l}<\alpha<\alpha_{l}$, where $\alpha_{l}<\pi$, which means that the antiparallelogram cannot encounter its two flat configurations. In practise, $\alpha_{l}$ is determined to avoid any collision. Here, $\alpha_{l}=140^{\circ}$. All other variables (orientation of the crossed bars, springs and cables lengths) can be expressed w.r.t $\alpha$. The dynamic model of the $\mathrm{X}$-joint is derived using the Lagrangian method. The mass and inertia of bars, motors, reducers and drums is taken into account in the dynamic model. The equation of motion can be written in the following form :

$$
M(\alpha) \ddot{\alpha}+C(\alpha) \dot{\alpha}^{2}+G(\alpha)=Z_{l}(\alpha) f_{l}+Z_{r}(\alpha) f_{r}
$$

Where $M$ is the inertia, $C$ the coefficient of Coriolis effect and $G$ the potential effects. The coefficients $Z_{l}$ and $Z_{r}$ link the forces $f_{1}$ and $f_{r}$ applied by the left and right cables to the generalized forces. Here they are defined by the torque applied by the cables on the instantaneous center of rotation of the X-joint (defined by the intersection point of the two crossed bars). The derivation and expression of the aforementioned quantities is not detailed here but can be found in [16].

\section{CONTROL OF A SINGLE JOINT (SIMULATION AND EXPERIMENTS)}

\section{A. Wrench feasible workspace}

The wrench feasible workspace (WFW) is the space of reachable positions in which the manipulator can balance a given set of external wrenches with bounded actuation forces. In our case, the actuation wrench is the set of forces that cables can apply and the external wrench is the forces due to gravity. Using the static part of the dynamic model, we compute all the orientations $\alpha$ such that $f_{\min }<$ $f_{l}<f_{\max }, f_{\min }<f_{r}<f_{\max }$ and in which the mechanism is in an equilibrium configuration [16], [19]. As shown in [16], [19], the X-shape mechanism has only one stable equilibrium configuration for a given set of applied forces. Moreover, there exists two symmetric unstable equilibrium configurations close to $-\pi$ and $\pi$. In our case, these unstable configurations are not reachable as they are beyond the joint limits.

\section{B. Experimental results}

For a desired trajectory $\left(\alpha_{d}, \dot{\alpha}_{d}, \ddot{\alpha}_{d}\right)$, a computed torque control with a proportional-integral-derivative (PID) correction is used. The forces $\left(f_{l}, f_{r}\right)$ are computed using (2):

$$
\begin{aligned}
& M(\alpha)\left(\ddot{\alpha}_{d}+k_{d}\left(\dot{\alpha}_{d}-\dot{\alpha}\right)+k_{p}\left(\alpha_{d}-\alpha\right)+k_{i} \int_{0}^{\tau}\left(\alpha_{d}-\alpha\right)(t) d t\right) \\
& +G(\alpha)=Z_{l}(\alpha) f_{l}+Z_{r}(\alpha) f_{r}
\end{aligned}
$$

For simplification reasons, the Coriolis effects are neglected (low velocities are assumed). The gains are calculated as explained in [22], namely, $k_{p}=3 \omega^{2}, k_{i}=3 \omega, k_{d}=\omega^{3}$, where $\omega$ is a function of the torque constant, the dielectric constant, the motor efficiency and its inertia. At each 
Following a trajectory

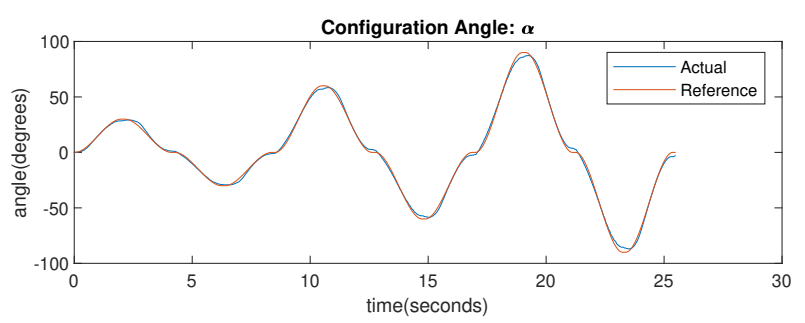

Error $=\alpha_{\text {ref }}{ }^{-\alpha}$, mean absolute error $=1.4768^{\circ}$ maximum absolute error $=4.5338^{\circ} \mathrm{SD}=1.0764^{\circ}$

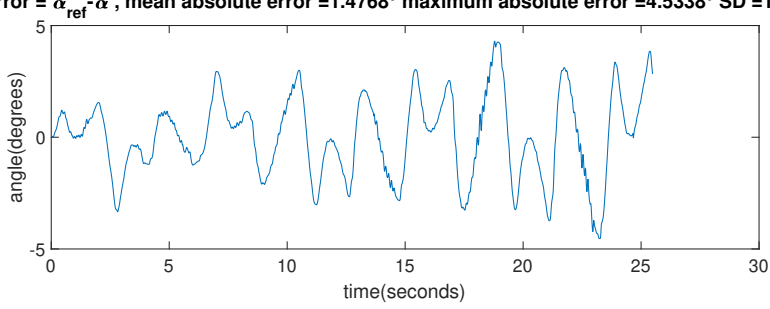

Fig. 3: Reference trajectory and real trajectory of the prototype, and real-time error on $\alpha$.

iteration, the actual orientation $\alpha$ of the mechanism is computed by measuring the motor positions with encoders. This encoder information is used to compute the effective cable lengths $l_{l}$ and $l_{r}$ and the corresponding value of $\alpha . \dot{\alpha}$ is computed numerically. Since in (2), there are two forces and one equation, an infinity of forces can be defined. A unique set of forces can be determined by additionally satisfying a desired stiffness as shown in [16]. In the experiments shown here, a simpler strategy was used so as to keep one of the forces to a fixed minimal value, $f_{\min }=3.5 \mathrm{~N}$. This strategy ensures that the cables are always in tension.

Since the mechanism is light, its own inertia is low as compared to the actuator inertia. The actuator inertia taking into account the full power train can be written w.r.t $\alpha$ as follows :

$$
M_{a}(\alpha)=\frac{I_{a}}{r_{d r u m}^{2}}\left[\left(\frac{\partial l_{l}}{\partial \alpha}\right)^{2}+\left(\frac{\partial l_{r}}{\partial \alpha}\right)^{2}\right]
$$

It is worth noting that $M_{a}$ strongly depends on $\alpha$. Thus, taking $M_{a}$ into account in (2) for the computation of $M(\alpha)$ is necessary to ensure a satisfactory trajectory tracking.

The results for a reference trajectory composed of three successive back-and-forth motions of increasing magnitude $\alpha_{d}=\left\{ \pm 30^{\circ}, \pm 60^{\circ}, \pm 90^{\circ}\right\}$ is shown in Fig. 3 and 4 , with $\omega=9 \mathrm{rd} / \mathrm{s}$ and $f_{\min }=3.5 \mathrm{~N}$. The mean absolute error for the whole trajectory is of $1.4768^{\circ}$, and the maximal value forces are smaller than $60 \mathrm{~N}$. The small oscillations in the forces are caused by the numerical computation of $\dot{\alpha}$ and by friction between the cable and shaft, but they do not impact the behavior of the joint.

These results validate the dynamic modelling of a single $\mathrm{X}$-joint, the performances of the dynamic control law, and the tuning of the PID controller, which will be used for the 3-DOF manipulator.
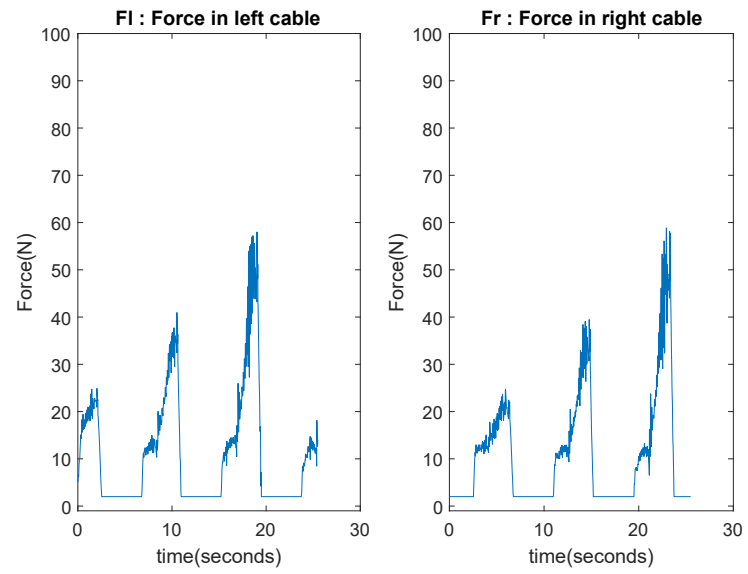

Fig. 4: Applied forces in the cables for the given trajectory.

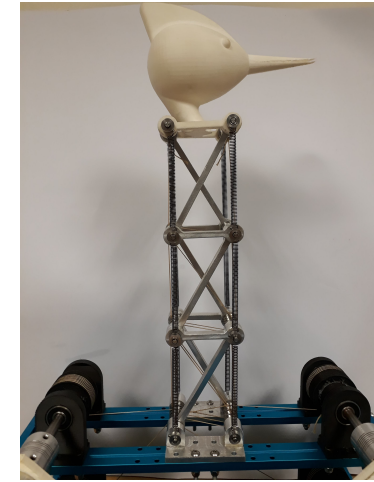

(a)

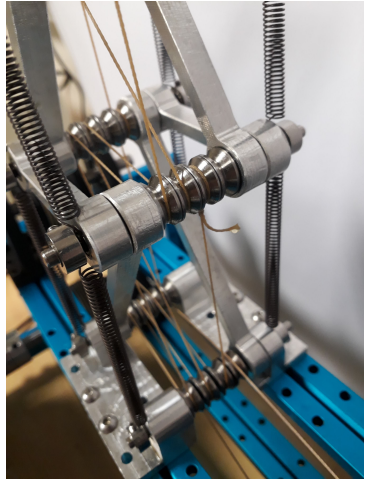

(b)
Fig. 5: 3-DoF manipulator (a) and focus on the actuation strategy and the cable routing (b). Each cable is run through pulleys and remains in one single motion plane. Routing of each cable is optimized to ensure tension and contact with pulleys in the full workspace.

\section{A 3-DOF MANIPULATOR WITH 3 X-JOINTS}

\section{A. Modelling}

Bird necks have a number of vertebrae varying from 10 to 26 depending on the species [14]. This means that the bird neck has a high kinematic redundancy. To start with, a model with three vertebrae is studied. Accordingly, a manipulator with three X-joints is analyzed. The manipulator is supposed to operate in a vertical plane for positioning tasks. The manipulator has thus a kinematic redundancy of 1 .

The three $\mathrm{X}$-joint are assembled in series, where joint 1 is fixed to the base and joint 3 carries the end-effector (here the bird head). Each mechanism $i$ is now equipped with 2 springs in parallel on each side (see Fig. 5). The bars are made of aluminum alloy and all parameters values are given in Tab. II.

\section{B. Actuation scheme}

The single $\mathrm{X}$-joint was actuated with two cables in an antagonist way. For the 3-DoF manipulator, several actuation 


\begin{tabular}{ccc}
\hline Parameter & Value & Unit \\
\hline \hline$b$ : length of base and top bars & 0.05 & $\mathrm{~m}$ \\
$L:$ length of crossed bars & 0.1 & $\mathrm{~m}$ \\
$m_{4}:$ mass of one top bar & 0.024 & $\mathrm{~kg}$ \\
$m_{2}, m_{3}:$ mass of one crossed bar & 0.042 & $\mathrm{~kg}$ \\
$k_{l 1}, k_{r 1}:$ spring stiffness of mechanism 1 & 600 & $\mathrm{~N} / \mathrm{m}$ \\
$k_{l 2}, k_{r 2}:$ spring stiffness of mechanism 2 & 200 & $\mathrm{~N} / \mathrm{m}$ \\
$k_{l 3}, k_{r 3}:$ spring stiffness of mechanism 3 & 200 & $\mathrm{~N} / \mathrm{m}$ \\
$l_{0}:$ spring free length (same for all springs) & 0.05 & $\mathrm{~m}$ \\
$I_{a}:$ Inertia of actuator & 0.0078 & $\mathrm{~kg} \cdot \mathrm{m}^{2}$ \\
$f_{\max }:$ Maximal force applied by cable & 155 & $\mathrm{~N}$ \\
\hline
\end{tabular}

TABLE II: 3-DoF Prototype data

strategies are possible. First, it is necessary to define an appropriate number of active cables and a suitable way of routing them from the motors to the mechanisms. Regarding the number of active cables, we propose to use four ones. This choice is a compromise between a minimal actuation ( 2 active cables) and a maximal one (6 active cables, 2 for each X-joint). With four cables, moreover, the three $\mathrm{X}$-joint can be fully controlled in position. Regarding the routing strategy of the cables, several schemes can be considered, including strut-routed [17] and side-routed [11], [23]. Here, a strut-routed strategy with one cable for each joint is used on the right side, while a side routed strategy with one single cable running along the three joints is used on the left side. This strategy is close to muscle scheme met in bird necks [24]. More specifically, one cable is run through the left sides of the three joints. This cable actuates the three Xjoints simultaneously. On the right sides, three cables are run through the joints, so that each single joint is actuated by one cable (see Fig. 6). This strut-routed actuation ensures that there is no dependency between the cables [23]. The path of each cable has been optimized to avoid any loss of contact between pulleys and cables. Each cable is attached to a drum linked to a motor shaft, and runs around the shafts up to the top bar shafts thanks to pulleys.

\section{Dynamic modeling}

The dynamic model of the 3-DOF manipulator can be written in vector form as follows:

$$
\mathbf{M}(\boldsymbol{\alpha}) \ddot{\boldsymbol{\alpha}}+\mathbf{C}(\boldsymbol{\alpha}) \dot{\boldsymbol{\alpha}}^{2}+\mathbf{G}(\boldsymbol{\alpha})=\mathbf{Z}_{\mathbf{l}}(\boldsymbol{\alpha}) \mathbf{f}_{\mathbf{l}}+\mathbf{Z}_{\mathbf{r}}(\boldsymbol{\alpha}) \mathbf{f}_{\mathbf{r}}
$$

For the chosen actuation scheme, $\mathbf{f}_{\mathbf{l}}=\left[f_{\text {long }}, f_{\text {long }}, f_{\text {long }}\right]^{\top}$ and $\mathbf{f}_{\mathbf{r}}=\left[f_{1}, f_{2}, f_{3}\right]^{\top}$, where $f_{\text {long }}$ is the force along the long cable on the left and $f_{1}, f_{2}, f_{3}$ are the forces along three cables on the right. All force components are greater than a minimal value $f_{\min } . \boldsymbol{\alpha}(t)$ is the vector of joint configurations, its $i^{\text {th }}$ component is denoted $\alpha_{i} . \mathbf{Z}_{\mathbf{l}}$ and $\mathbf{Z}_{\mathbf{r}}$ are diagonal matrices, where the $i^{t h}$ entry depends only on $\alpha_{i}$ [25].

\section{Wrench Feasible Workspace}

The WFW is computed by discretization of the joint space. As previously, the joint limits are $-140^{\circ}<\alpha_{i}<-140^{\circ}$, $i=1,2,3$. If the forces required to keep the manipulator in equilibrium are within their bounds, the configuration is in the WFW. The static model is used to compute the forces

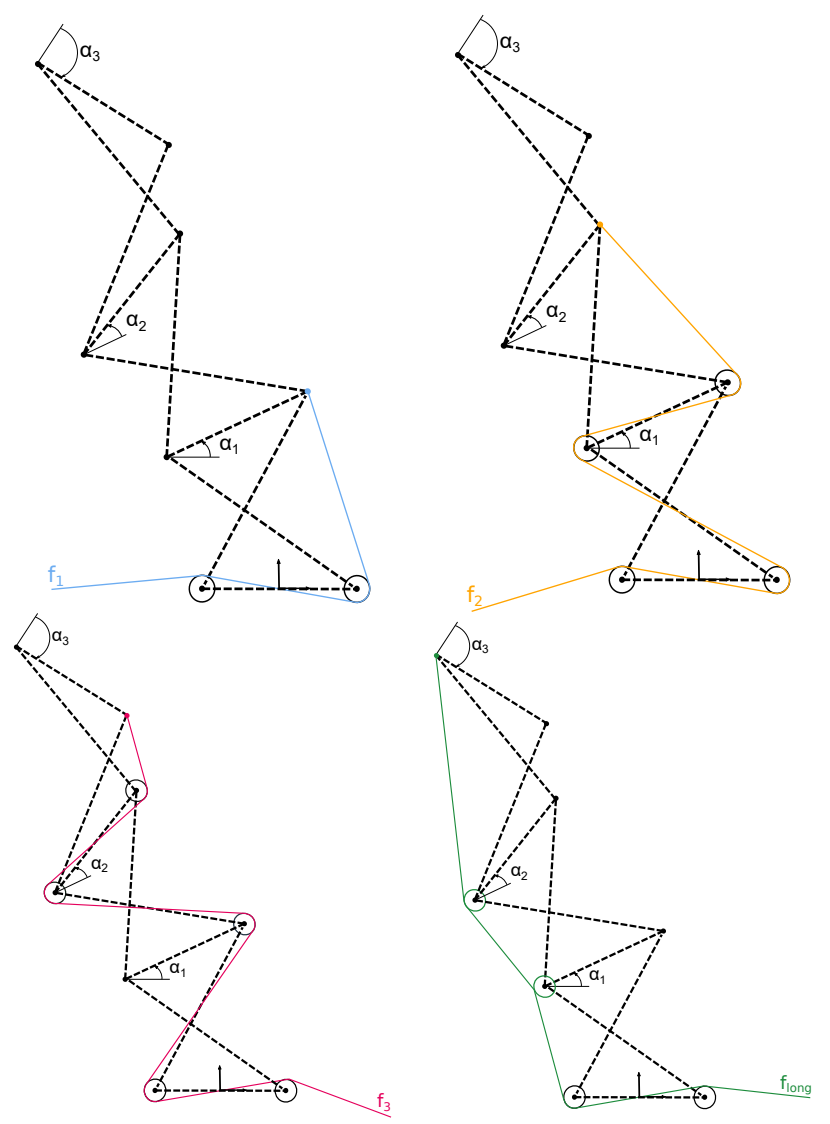

Fig. 6: Actuation scheme for the three-DoF manipulator in an arbitrary configuration. Bars are in dotted lines and cables in plain lines. Each cable is on its own layer of pulleys.

for a given joint configuration, which can be set as:

$$
\mathbf{G}(\boldsymbol{\alpha})=\mathbf{Z}_{\mathbf{l}}(\boldsymbol{\alpha}) \mathbf{f}_{\mathbf{l}}+\mathbf{Z}_{\mathbf{r}}(\boldsymbol{\alpha}) \mathbf{f}_{\mathbf{r}}
$$

The WFW is first calculated for the four-cable actuation scheme used for the prototype. To compute the forces in (5), the following method is used. Let $g_{i}$ be $i^{\text {th }}$ component of $\mathbf{G}(\alpha)$. For each joint $i$, the applied forces must satisfy:

$$
g_{i}=Z_{l i}\left(\alpha_{i}\right) f_{\text {long }}+Z_{r i}\left(\alpha_{i}\right) f_{i}
$$

where $Z_{l i}\left(\alpha_{i}\right)>0$ and $Z_{r i}\left(\alpha_{i}\right)<0$ [25].

Since in the three linear equations (6), there are four unknown variables $f_{1}, f_{2}, f_{3}, f_{\text {long }}$, an infinite number of actuation forces exist. The solution minimizing the antagonistic forces is chosen, while insuring that all forces $f_{1}, f_{2}, f_{3}, f_{\text {long }}$ are greater than $f_{\min }$. The minimal value of $f_{\text {long }}$ is calculated to satisfy the four constraints independently. Accordingly, its value is defined as the maximum of the minimal values:

$$
f_{\text {long }}=\max _{i \in\{1, \ldots, 3\}}\left(\frac{g_{i}-Z_{r i}\left(\alpha_{i}\right) f_{\min }}{Z_{l i}\left(\alpha_{i}\right)}, f_{\min }\right)
$$

Then, $f_{i}$ is computed for each joint $i$ as a function of $f_{\text {long }}$ :

$$
f_{i}=\frac{g_{i}-Z_{l i}\left(\alpha_{i}\right) f_{\text {long }}}{Z_{r i}\left(\alpha_{i}\right)}
$$




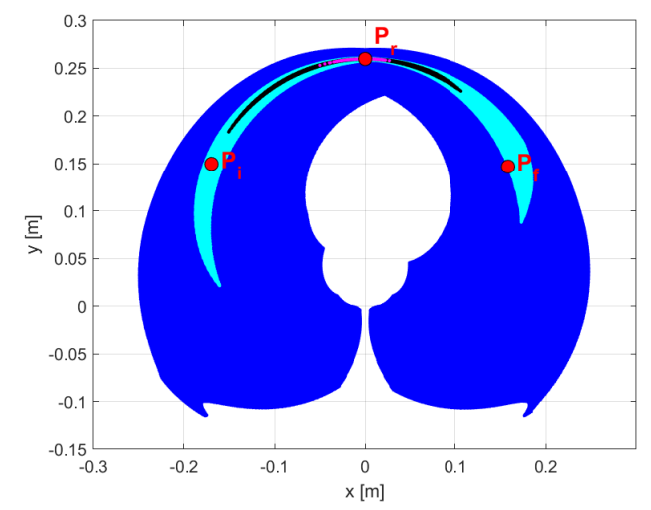

Fig. 7: In blue, the WFW of the manipulator actuated with four cables. In the cyan, black and magenta regions the sum of the applied forces are lower than $20 \mathrm{~N}, 5 \mathrm{~N}$ and $1 \mathrm{~N}$, respectively.

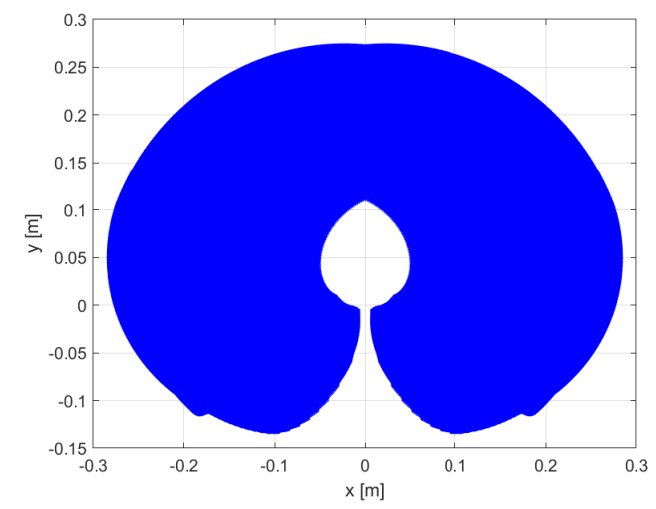

Fig. 8: WFW of the manipulator actuated with six cables.

The resulting WFW, limited by the constraint that each force must be lower that $f_{\max }$, is shown in Fig. 7. Note that it is not symmetrical due to the asymmetrical actuation strategy.

To evaluate the efficiency of the chosen actuation scheme, the WFW is also computed for a full actuation strategy (six cables, two antagonistic cables at each joint). In this case, the three equations in (5) can be solved independently, and each equation has an infinite number of solutions. Since each force must be greater than $f_{\text {min }}$, the solution minimizing the sum of the forces is obtained as follows:

$$
\begin{array}{lll}
f_{l i}=f_{\text {min }}, & f_{r i}=\frac{g_{i}-Z_{l i}\left(\alpha_{i}\right) f_{\text {min }}}{Z_{r i}\left(\alpha_{i}\right)} \quad, \text { if } g_{i} \leq g_{m i} \\
f_{r i}=f_{\text {min }}, & f_{l i}=\frac{g_{i}-Z_{r i}\left(\alpha_{i}\right) f_{\text {min }}}{Z_{l i}\left(\alpha_{i}\right)} \quad, \text { if } g_{i}>g_{m i}
\end{array}
$$

where $g_{m i}=\left(Z_{l i}\left(\alpha_{i}\right)+Z_{r i}\left(\alpha_{i}\right)\right) f_{\text {min }}$ and $f_{l i}, f_{r i}$ are the forces applied on the left and right side of joint $i$, respectively. The resulting WFW is shown in Fig. 8. As compared to the WFW with four cables, the difference in size is not so large.

\section{E. Equilibrium configurations at rest}

The equilibrium configurations at rest (i.e. with no actuation forces) satisfies:

$$
\mathbf{G}(\boldsymbol{\alpha})=\mathbf{0}
$$

Equation (9) is difficult to solve algebraically but the upright configuration where $\alpha_{1}=\alpha_{2}=\alpha_{3}=0$ is found to be an equilibrium solution at rest as expected for symmetrical springs. To verify if there is no other equilibrium configurations at rest in the WFW, we have calculated those parts of the WFW where the sum of the actuation forces is lower than a threshold chosen as $1 \mathrm{~N}$ (assuming here $f_{\min }=0 \mathrm{~N}$ ). As shown in Fig. 7 , there is a small region around the upright configuration (shown in red). In addition to the equilibrium configurations at rest, it is interesting to find the configurations associated with low actuation forces. Regions of the WFW where the sum of the actuation forces is lower than $5 \mathrm{~N}$ and $20 \mathrm{~N}$ are depicted in black and cyan, respectively (see Fig. 7).

\section{F. Control}

Let $\boldsymbol{\alpha}_{d}(t)$ be the desired joint trajectory. A computed torque control with PID gains is used as for the single $\mathrm{X}$-joint. The desired torque at time $t$ is defined using the dynamic model (with the centrifugal and Coriolis effects neglected as above):

$$
\mathbf{c}_{d}(t)=\mathbf{M}(\boldsymbol{\alpha}(t)) \mathbf{w}(t)+\mathbf{G}(\boldsymbol{\alpha}(t))
$$

with

$$
\mathbf{w}(t)=\ddot{\boldsymbol{\alpha}}_{d}(t)+k_{v} \dot{\mathbf{e}}(t)+k_{p} \mathbf{e}(t)+k_{i} \int_{t^{\prime}=0}^{t}\left(\mathbf{e}\left(t^{\prime}\right)\right) d t
$$

Once the desired torque is defined, the actuation forces are calculated using (7) and (8) where $g_{i}$ is replaced with the $i^{t h}$ component of $\mathbf{c}_{d}(t)$. If a force is found to be out of its bounds, it is updated with its nearest bound.

\section{G. Trajectory planning}

Let us define a task where the manipulator must move between three points in the Cartesian space. Starting from the upright position $P_{r}$ (equilibrium configuration at rest), the manipulator moves successively to two positions $P_{i}$ and $P_{f}$ and finally comes back to $P_{r}$ (points $P_{r}, P_{i}$ and $P_{f}$ are shown on Fig. 7). Since the control is defined in the joint space, a joint trajectory must be calculated. The kinematic redundancy is solved by minimizing the actuated forces using the static model. First of all, the optimal inverse solutions at $P_{r}, P_{i}$ and $P_{f}$ are calculated by solving the inverse kinematics while minimizing the sum of the actuated forces as follows:

$$
\begin{array}{ll} 
& \min _{\boldsymbol{\alpha}}\left(f_{1}+f_{2}+f_{3}+f_{\text {long }}\right) \\
\text { s.t. } & P=F G M(\boldsymbol{\alpha}), \\
& \mathbf{G}(\boldsymbol{\alpha})=\mathbf{Z}_{\mathbf{l}}(\boldsymbol{\alpha}) \mathbf{f}_{\mathbf{l}}+\mathbf{Z}_{\mathbf{r}}(\boldsymbol{\alpha}) \mathbf{f}_{\mathbf{r}} .
\end{array}
$$

where $F G M$ is the forward geometric model, and $P$ is $P_{r}, P_{i}$ or $P_{f}$. The resulting joint configurations are denoted $\boldsymbol{\alpha}_{r}, \boldsymbol{\alpha}_{i}$ and $\boldsymbol{\alpha}_{f}$. With no surprise, $\boldsymbol{\alpha}_{\boldsymbol{r}}=[0,0,0]^{T}$. The optimal joint 


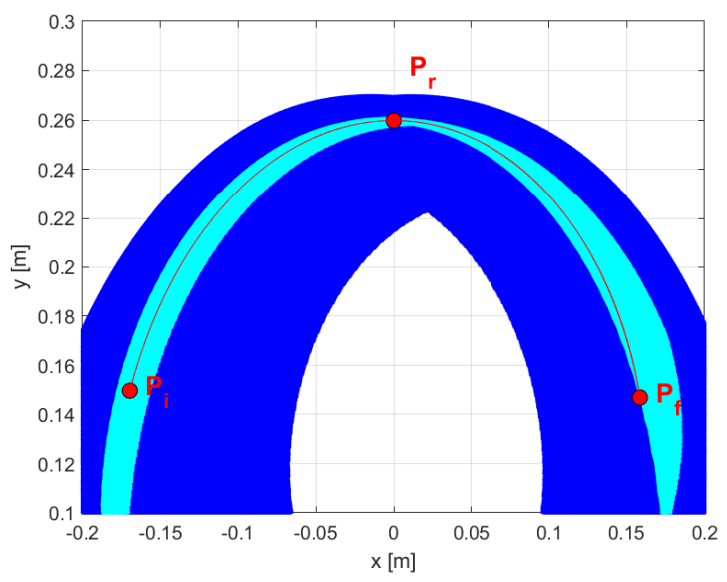

Fig. 9: Optimal path in the Cartesian space with minimal actuation forces. The cyan color shows the area of the WFW reachable with $C<20 \mathrm{~N}$.

trajectory is then obtained using an $A^{*}$ algorithm associated with the cost function $C$ below :

$$
C=\sum_{j=1, N}\left(f_{1}+f_{2}+f_{3}+f_{\text {long }}\right)
$$

where $N$ is the number of points along the discretized path. A polynomial function is used to link the $N$ points using a normalized curvilinear abscissa $p$. As a stop is imposed at each point, a cycloid function is finally used to define the timing law $p(t)$ along the path. The resulting joint trajectory is shown in Fig. 10. A $2 \mathrm{~s}$ stop is imposed at each point. The motion between $P_{r}, P_{i}$ and between $P_{f}, P_{r}$ is performed in $2 \mathrm{~s}$. The motion between $P_{i}$ and $P_{f}$ is performed in $4 \mathrm{~s}$. In the future, faster motions will be experimented using the full dynamic model instead of the static model for the trajectory optimization. It is worth noting that the optimal path between $P_{i}$ and $P_{f}$ passes through $P_{r}$ whereas this was not prescribed. This is due to the cost function that tends to minimize the actuation forces during motion. Moreover, the optimal path in the Cartesian space remains in the area with low forces as shown in Fig. 9.

\section{H. Experiments}

As for the single joint experiments, the encoder information is used to compute the effective cable length $l_{r i}$ at each iteration (only on the right here because the long cable does not provide additional information) and the corresponding values of $\boldsymbol{\alpha} . \dot{\boldsymbol{\alpha}}$ are computed numerically.

The results for the optimized reference trajectory obtained in the previous section is shown in Fig. 10 and 11, with $\omega=9 \mathrm{rd} / \mathrm{s}$ and $f_{\min }=3.5 \mathrm{~N}$ to ensure a minimal tension in the cable. The tracking error is acceptable. The absolute mean error is around $0.6^{\circ}$, while the maximum error is around $6^{\circ}$. Since the actuation scheme is asymmetrical, the force behavior depends on the direction of motion. When the manipulator moves to the left, $f_{\text {long }}$ is high as compared to $f_{1}$, $f_{2}, f_{3}$ and conversely. The actuation forces are not too high even if higher than their expected values from simulation.
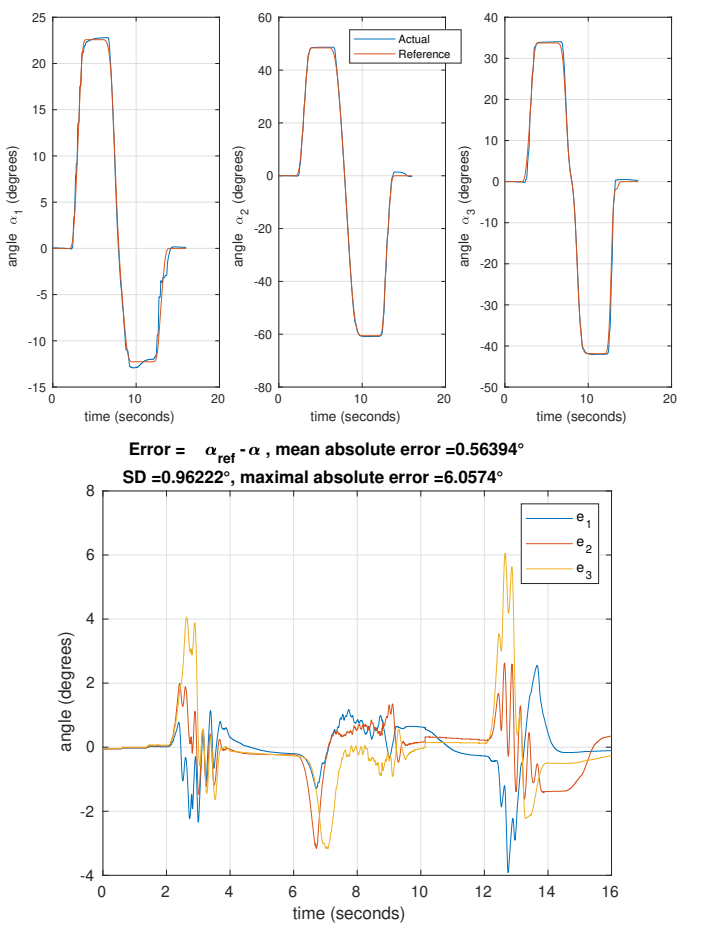

Fig. 10: Reference trajectory and real trajectory of the prototype, and real-time error on $\alpha$.

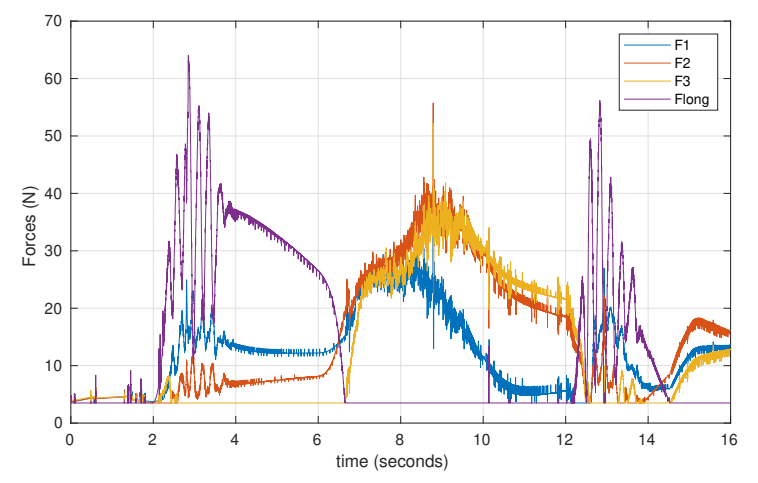

Fig. 11: Applied forces in the cables for the given trajectory.

The difference can be explained by two main factors. First, we have taken $f_{\min }=3.5 \mathrm{~N}$ in the experiments to ensure a minimal cable tension while $f_{\min }=0 \mathrm{~N}$ in simulation. Second, friction effects have been neglected in simulation. The main oscillations on $f_{\text {long }}$ are due to the friction of the long cable due to the loop at each pulley (capstan effect). A more efficient routing strategy of the long cable using a double pulley will be realized in the near future.

\section{CONCLUSIONS AND FUTURE WORK}

A new concept of light-weight manipulator with $\mathrm{X}$-joints in series driven by cables has been presented and studied. This concept has been validated on a single-joint testbed and on a 3-DOF prototype manipulator moving along a trajectory that minimizes the actuation forces. Although the inertia of the manipulator itself is low, that of the full power 
train is significant and turned out to have a highly variable contribution in the inertia matrix of the dynamic model. Accordingly, a dynamic control law instead of a simple PID controller has been used. In future work, the kinematic redundancy will be exploited with a Cartesian space control to move the manipulator among obstacles by shaping around them rather than trying to avoid these latter. Experiments will be conducted on a prototype with ten $\mathrm{X}$-joints.

\section{REFERENCES}

[1] F. R. Buckminster, "Tensile-integrity structures," Nov. 13 1962. US Patent 3,063,521.

[2] R. Motro, "Tensegrity systems: the state of the art," International journal of space structures, vol. 7, pp. 75-83, June 1992.

[3] R. E. Skelton and M. C. de Oliveira, Tensegrity systems, vol. 1. Boston, MA: Springer, 2009.

[4] S. M. Levin, "The tensegrity-truss as a model for spine mechanics: biotensegrity," Journal of mechanics in medicine and biology, vol. 2, no. 03n04, pp. 375-388, 2002.

[5] M. Arsenault and C. M. Gosselin, "Kinematic, static and dynamic analysis of a planar 2-dof tensegrity mechanism," Mechanism and Machine Theory, vol. 41, pp. 1072-1089, September 2006.

[6] C. D. Crane, J. Bayat, V. Vikas, and R. Roberts, "Kinematic analysis of a planar tensegrity mechanism with pre-stressed springs," in Advances in Robot Kinematics: analysis and design (L. J. and W. P., eds.), pp. 419-427, Dordrecht, Germany: Springer, 2008.

[7] P. Wenger and D. Chablat, "Kinetostatic analysis and solution classification of a planar tensegrity mechanism," in Proc. th Int.l Workshop on Computational Kinematics, May 22-24, 2017, Poitiers, France, pp. 422-431, Springer, May 2017.

[8] J. Rieffel and J.-B. Mouret, "Adaptive and resilient soft tensegrity robots," Soft robotics, vol. 5, pp. 318-329, June 2018.

[9] V. Böhm, T. Kaufhold, F. Schale, and K. Zimmermann, "Spherical mobile robot based on a tensegrity structure with curved compressed members," in 2016 IEEE Int. Conf. on Adv. Intel. Mechatronics (AIM), July 12-15, 2016, Banff, Canada, pp. 1509-1514, IEEE, 2016.

[10] M. Vespignani, J. M. Friesen, V. SunSpiral, and J. Bruce, "Design of superball v2, a compliant tensegrity robot for absorbing large impacts," in 2018 IEEE/RSJ Int. Conf. on Intel. Robots and Systems (IROS), October 1-5, 2018, Madrid, Spain, pp. 2865-2871.

[11] D. L. Bakker, D. Matsuura, Y. Takeda, and J. L. Herder, "Design of an environmentally interactive continuum manipulator," in Proc. 14th IFToMM World Congress in Mechanism and Machine Science, October 25-30, 2015, Taipei, Taiwan, pp. 327 - 336, 2015.

[12] S. Chen and M. Arsenault, "Analytical computation of the actuator and cartesian workspace boundaries for a planar 2-degree-offreedom translational tensegrity mechanism," Journal of Mechanisms and Robotics, vol. 4, p. 011010, February 2012.

[13] K. D. Snelson, "Continuous tension, discontinuous compression structures," Feb. 16 1965. US Patent 3,169,611.

[14] G. Zweers, R. Bout, and J. Heidweiller, "Motor organization of the avian head-neck system," in Perception and motor control in birds : An ecological approach (M. N. O. Davies and P. R. Green, eds.), pp. 201-221, Dordrecht, Germany: Springer, 1994.

[15] Q. Boehler, I. Charpentier, M. S. Vedrines, and P. Renaud, "Definition and computation of tensegrity mechanism workspace," Journal of Mechanisms and Robotics, vol. 7, p. 044502, Nov 2015.

[16] A. Van Riesen, M. Furet, C. Chevallereau, and P. Wenger, "Dynamic analysis and control of an antagonistically actuated tensegrity mechanism," in Proc. 22nd CISM IFToMM Symp. on Robot Design, Dynamics and Control (ROMANSY), June 25-28, 2018, Rennes, France, pp. 481-490, Springer, 2018.

[17] J. Aldrich and R. Skelton, "Time-energy optimal control of hyperactuated mechanical systems with geometric path constraints," in Proc. 4th IEEE Conf. on Decision and Control, December 15, 2005, Seville, Spain, pp. 8246-8253, IEEE, 2005.

[18] M. Arsenault and C. M. Gosselin, "Kinematic and static analysis of a planar modular 2-dof tensegrity mechanism," in Proc. 006 IEEE Int. Conf. on Ro.and Aut. (ICRA), May 15-19, 2006, Orlando, FL, USA, pp. 4193-4198, IEEE, 2006.
[19] M. Furet, A. Van Riesen, C. Chevallereau, and P. Wenger, "Optimal design of tensegrity mechanisms used in a bird neck model," in Proc. o7th European Conf. on Mechanism Science (EuCoMeS), September 4 - 6, 2018, Aachen, Germany, pp. 365-375, Springer, 2018.

[20] M. Furet, M. Lettl, and P. Wenger, "Kinematic analysis of planar tensegrity 2-x manipulators," in Proc. 6th Int. Symp. on Advances in Robot Kinematics (ARK), July 1-5, Bologna, Italia, pp. 153-160, Springer, 2018.

[21] M. Furet and P. Wenger, "Workspace and cuspidality analysis of a 2-x planar manipulator," in Proc. 4th IFToMM Symp. on Mechanism Design for Robotics (MEDER), September 11 - 13, 2018, Udine, Italy, pp. 110-117, Springer, 2018.

[22] S. Caro, D. Chablat, P. Lemoine, and P. Wenger, "Kinematic analysis and trajectory planning of the orthoglide 5-axis," in Proc. Int. Design Eng. Technical Conf. and Comp. and Inf. in Engineering Conference, August 2-5, 2015, Boston, USA, ASME, 20159.

[23] M. Furet and P. Wenger, "Kinetostatic analysis and actuation strategy of a planar tensegrity 2-X manipulator," Journal of Mechanisms and Robotics, pp. 1-19, July 2019.

[24] C. Bohmer, M. Furet, B. Fasquelle, P. Wenger, D. Chablat, C. Chevallereau, and A. Abourachid, "Combining precision and power to maximize performance: a case study of the woodpecker's neck," in 44ème congrès de la Société de Biomécanique, Poitiers, France, 2019.

[25] B. Fasquelle, M. Furet, C. Chevallereau, and P. Wenger, "Dynamic modeling and control of a tensegrity manipulator mimicking a bird neck," in Proc. 15th IFToMM World Congress on Mech. and Mach. Science, June 30-July 4, 2019, Krakow, Poland, pp. 2087-2097, Springer, 2019. 